\title{
Interventions to modify physical activity in patients with COPD: a systematic review
}

\author{
Leandro Cruz Mantoani ${ }^{1}$, Noah Rubio ${ }^{1}$, Brian McKinstry ${ }^{2,3}$, William MacNee ${ }^{1}$ \\ and Roberto A. Rabinovich ${ }^{1}$ \\ Affiliations: \\ ${ }^{1}$ ELEGI and COLT Laboratories, The Queen's Medical Research Institute, The University of Edinburgh, \\ Edinburgh, UK. \\ ${ }^{2}$ Edinburgh Health Services Research Unit/Edinburgh Clinical Trials Unit, Western General Hospital, \\ Edinburgh, UK. \\ ${ }^{3}$ Telescot Research Programme, Usher Institute of Population Health Sciences and Informatics, The University \\ of Edinburgh, Edinburgh, UK.
}

\section{Correspondence:}

Roberto Rabinovich, ELEGI and COLT Laboratories, The Queen's Medical Research Institute, 47 Little France Crescent, Edinburgh, EH16 4TJ, UK.

E-mail: Roberto.Rabinovich@ed.ac.uk

ABSTRACT The broad range of interventions to increase physical activity (PA) in patients with chronic obstructive pulmonary disease (COPD) has not been systematically assessed. We aimed to perform a systematic review of the interventional studies that have assessed PA as an outcome in patients with COPD.

A systematic search in five different databases (Medline, Embase, PsycINFO, CINAHL and Web of Science) was performed in March 2015. Two independent reviewers analysed the studies against the inclusion criteria (COPD defined by spirometry; prospective, randomised/nonrandomised studies, cohort and experimental studies with interventions using PA as an outcome), extracted the data and assessed the quality of evidence.

60 studies were included. Seven intervention groups were identified. PA counselling increased PA levels in COPD, especially when combined with coaching. 13 studies showed positive effects of pulmonary rehabilitation (PR) on PA, while seven studies showed no changes. All three PR programmes $>12$ weeks in duration increased PA. Overall, the quality of evidence was graded as very low.

Interventions focusing specifically on increasing PA, and longer PR programmes, may have greater impacts on PA in COPD. Well-designed clinical trials with objective assessment of PA in COPD patients are needed.

@ERSpublications

Long-lasting pulmonary rehabilitation and physical activity coaching programmes increase physical activity in COPD http://ow.ly/Zi8T2

Editorial comment in: Eur Respir J 2016; 48: 14-17.

This article has supplementary material available from erj.ersjournals.com

Received: Oct 212015 | Accepted after revision: March 042016 | First published online: April 212016

Support statement: L.C. Mantoani is funded by an EPSRC/Philips Healthcare grant and the College of Medicine and Veterinary Medicine of The University of Edinburgh.

Conflict of interest: Disclosures can be found alongside this article at erj.ersjournals.com

Copyright CERS 2016 


\section{Introduction}

Assessment of physical activity (PA) in daily life as an outcome has generated growing scientific interest in recent years, especially in chronic respiratory diseases [1,2], due to its relationship with mortality [3]. It is well known that preserved PA levels may delay or even prevent the appearance of chronic diseases $[4,5]$. Patients with chronic obstructive pulmonary disease (COPD) are characterised by a sedentary lifestyle and reduced PA levels [6]. This inactivity is an independent predictor of the risk of hospitalisations due to acute exacerbations and early mortality in this population $[1,2]$. Therefore, improving PA levels has been considered a key component in the management of patients with COPD.

Pulmonary rehabilitation (PR) is the most successful intervention aimed at improving symptoms (dyspnoea, muscle fatigue), exercise capacity, health-related quality of life, healthcare utilisation and costs in individuals with chronic respiratory disease [7]. However, the translation of the improvements in exercise capacity into increments in PA levels is less evident and still controversial $[8,9]$. The ability to increase PA levels using other therapeutic strategies, such as PA counselling [10, 11], nutritional supplementation [12], long-term oxygen therapy (LTOT) [13] and bronchodilators [13, 14], has also been explored in COPD and the effects to date are variable. These different types of interventions were cited in two recent reviews $[15,16]$ and comprise the majority of the interventions able to change PA levels in patients with COPD. Therefore, we chose to use them as part of the search strategy of the present systematic review, in order to summarise the wide range of interventions that could increase PA in this population. Currently, it is still unclear which is the best strategy to increase PA levels in patients with COPD.

Recent systematic literature reviews have addressed the determinants and outcomes of PA in COPD [15], investigated the effects of PA counselling on PA and health-related outcomes in chronic disease [16], and outlined the components of PA interventions other than PR aimed at increasing PA in patients with COPD [17]. These well-designed and well-conducted systematic reviews have generated important scientific knowledge. However, there has been no systematic summary of the interventions aimed at modifying PA levels in patients with COPD. Thus, the aims of the present study were to perform a systematic review of the interventional studies that have assessed PA as an outcome in patients with COPD.

\section{Methods}

Data sources and search strategy

This systematic review followed the handbook of the Centre for Reviews and Dissemination [18] and the PRISMA (Preferred Reporting Items for Systematic Reviews and Meta-Analyses) guidelines [19] for reporting systematic review analysis. A computerised literature search was performed in the following databases in March 2015 (date of last update): Medline/PubMed, Embase, PsycINFO, CINAHL and Web of Science. We performed some pre-piloted searches prior to the final search strategy, based on two recently published systematic reviews on a related topic $[15,16]$. We also performed a hand search of the studies based on the bibliographic references of the included articles and PubMed's "related articles" search filters.

Our search strategy included a wide range of modalities with variable levels of evidence for modifying PA levels in COPD (e.g. exercise training, PA counselling, nutritional supplementation, LTOT and bronchodilators) (see online supplementary material for details) [15]. Bibliographic details of all articles from the different databases were stored in a reference managing software file (EndNote X7; Thomson Reuters, New York, NY, USA). We used a simple symbol system in EndNote to record reviewers' decisions on inclusion or exclusion of each article. More details about the protocol of the present systematic review can be found in the supplementary material.

\section{Eligibility and exclusion criteria}

Eligible studies were included if they fulfilled the following a priori defined criteria. 1) Population/ participants: patients with COPD defined by spirometry (i.e. post-bronchodilator forced expiratory volume in $1 \mathrm{~s}\left(\mathrm{FEV}_{1}\right)$ /forced vital capacity <0.7). 2) Study design: i) prospective longitudinal studies; ii) randomised and nonrandomised clinical trials, both arms (intervention plus control) if the outcome was PA; iii) cohort studies; and iv) experimental or pilot studies of any type of intervention targeting PA in patients with COPD. 3) Studies with interventions that have assessed PA as an outcome, defined as "any bodily movement produced by skeletal muscles that results in energy expenditure" [20]. Exclusion criteria were articles in a non-English language, review articles, notes, editorials, qualitative studies and scientific congress abstracts.

\section{Study selection}

Two independent reviewers (L.C. Mantoani and N. Rubio) screened the titles and abstracts of every citation against the inclusion criteria. The reviewers' decisions on including or excluding all the retrieved articles were recorded in the EndNote file. Potentially eligible articles were highlighted and retrieved for full text evaluation. The same independent reviewers assessed the remaining articles and made a decision on 
inclusion or exclusion based on the eligibility criteria. Any disagreements between the reviewers were usually resolved by consensus. Persistent disagreements were resolved by a third independent reviewer (R.A. Rabinovich). We recorded the bibliographic details of all excluded studies with the specific reasons for excluding them from the final analysis (see supplementary material for details).

\section{Data extraction and quality assessment}

From each included study, we extracted and recorded the following information in an Excel file: authors, journal, year of publication, study design, setting, sample size, blindness, patient characteristics (sex, age, FEV1 and body mass index (BMI)), interventions (type, frequency, intensity and duration), PA measurements, outcomes and results.

We used the GRADE (Grading of Recommendations Assessment, Development and Evaluation) approach $[21,22]$ to assess the quality of the included studies. The GRADE system has been used by many international organisations and is a transparent approach created to help systematic reviewers and guideline authors to rate the quality of the evidence and the strength of recommendations [21]. The GRADE approach assesses the overall quality of the studies and provides a score for the body of evidence instead of a score for each study. Therefore, our judgement on the quality of the evidence across the included studies was based on each group of studies of the different interventions to modify PA levels in patients with COPD (i.e. exercise training category). Two reviewers (L.C. Mantoani and R.A. Rabinovich) assessed the quality of evidence and categorised it accordingly (high, moderate, low or very low) [21, 22]. The body of the evidence for each identified intervention category started with the high quality level on GRADE (high confidence between true and estimated effect) and was downgraded for specific reasons [21, 22]. Therefore, very low quality evidence is the lowest score for a body of evidence. This means there is very little confidence in the estimated effect and more studies are very likely to change the estimate. More details on how we used the GRADE system to judge the quality of included studies can be found in the supplementary material.

\section{Analysis and data synthesis}

As expected, there was marked heterogeneity among included studies, with a wide range of both different interventions to modify and different methods to assess PA, which precluded a meta-analysis. Findings were summarised using harvest plots $[23,24]$ of the retrieved eligible studies. Although it was not possible to statistically summarise the results of the included studies, we have calculated the average percentage change in PA levels according to each intervention's category. This was calculated using the percentage of change relative to baseline levels in each measured variable (subjective or objective) of each study, then calculating the average change (as a percentage) considering each study within every intervention category.

\section{Results}

A flow diagram of the study is shown in figure 1. In total, 2495 articles were retrieved. After removing the duplicates from the different database searches, 2091 articles were analysed. 1946 were excluded based on the titles and abstract screening, leaving 145 articles considered as potentially eligible for full text analysis. An additional 22 articles were identified from among the references of these 145 articles and from the related articles search filter in PubMed as suitable for full text assessment. After excluding 107 of these studies for the reasons specified in figure 1, 60 articles were considered for data extraction in this systematic review.

\section{Summary of studies}

From the 60 studies included in this systematic review, 33 were randomised controlled trials (RCTs), 18 were non-RCTs and nine were experimental or pilot studies (six of them with a randomisation). Table 1 shows the reference details, study design, number of participants, type of intervention and methods for measuring PA in each of these studies. Additional information on the patients' characteristics, performed interventions and methods of measuring PA for the 60 included studies is presented in table S1 of the supplementary material. Seven types of intervention with the potential to increase PA levels in patients with COPD were identified: PA counselling, nutritional supplementation (dietary intervention), LTOT, bronchodilator, nocturnal noninvasive ventilation (NIV), neuromuscular electrical stimulation (NMES) and exercise training (including PR programmes and other exercise interventions).

30 of the included studies used exercise training as the main intervention to modify PA levels (20 of them with PR alone). 14 studies used PA counselling, four exploited the benefits of nutritional supplementation, three used LTOT, six used bronchodilators, two used nocturnal NIV and one study used NMES as its main intervention. Seven studies combined two different types of interventions (e.g. PR plus PA counselling).

38 studies assessed the level of daily PA with objective measurements (accelerometers, multisensors and/or pedometers), while 27 studies measured PA levels using subjective assessments (questionnaires and/or scales). 


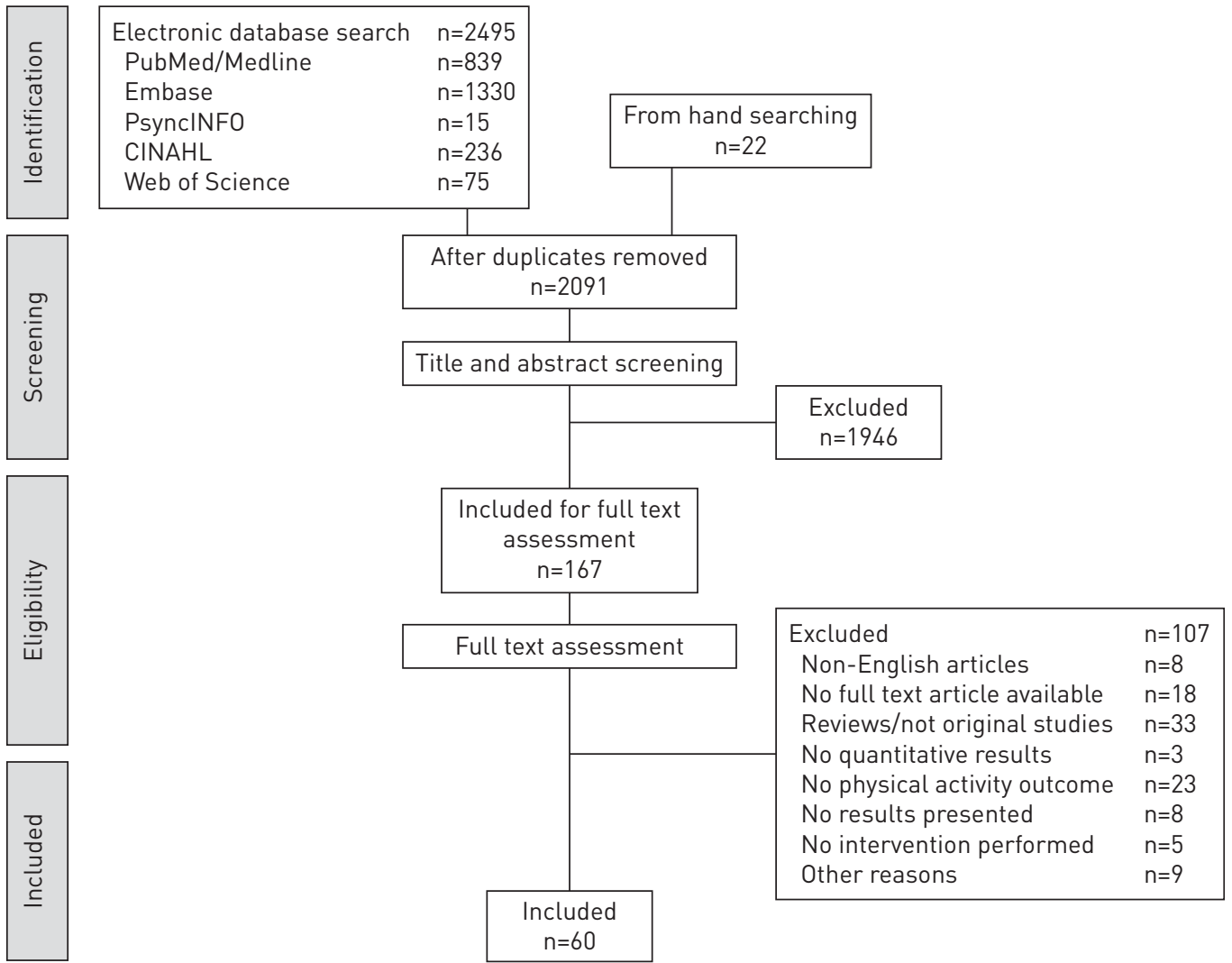

FIGURE 1 Flow diagram of the systematic review.

Four studies used both objective and subjective methods of measuring PA. More recent publications (from 2008 to date) chose more objective than subjective tools to measure daily PA levels in patients with COPD.

Overall, the quality of evidence across interventional studies to modify PA in patients with COPD was graded as very low (table 2). Serious imprecision and inconsistency across studies, as well as risk of bias (lack of blinding, concealment and/or randomisation process) and strongly suspected publication bias (studies with small sample size and absence of published negative results) were the main causes to downgrade the score for the quality of evidence. Directness was found across studies considering population, interventions and outcomes, regardless of intervention category.

\section{Harvest plots and intervention characteristics}

PA counselling

This category included three studies in which only advice on PA (PA advice) was given and 11 studies in which a coaching programme towards a more active life (PA coaching) was used with regular activity monitoring and incentive (figure 2a). Two studies in each category combined the PA intervention with exercise training. Almost half of the studies assessing the effect of PA counselling on PA levels were RCTs and most of these studies used objective tools to assess PA levels. Most studies were of a small sample size, included patients with moderate to very severe airflow limitation and had intervention periods of $>8$ weeks. The harvest plot shows that the most common intervention was PA coaching with the use of an activity monitor (figure $2 \mathrm{a}$ ). The majority of the interventions (11 out of 14 ) increased PA levels, especially when they included objective activity monitoring. More details about the use of harvest plots in the present systematic review can be found in the supplementary material.

\section{Nutritional supplementation}

All the studies using nutritional supplementation were RCTs and $75 \%$ of the studies used objective tools to assess PA levels. All studies included patients with moderate to very severe airflow limitation with low BMI $\left(\leqslant 20 \mathrm{~kg} \cdot \mathrm{m}^{-2}\right)$. All studies provided nutritional supplementation to the patients for a minimum period of 8 weeks. $75 \%$ of the studies showed a positive impact on the levels of daily activities after treatment (figure 2b). 
TABLE 1 Reference details and study characteristics of the 60 studies included in the present systematic review

\begin{tabular}{|c|c|c|c|c|}
\hline First author [ref.] & Year & Study design & $\begin{array}{c}\text { Subjects } \\
n\end{array}$ & Intervention type \\
\hline AltenBuRg [25] & 2015 & RCT & 155 & PA counselling \\
\hline BARBERAN-GARCIA [26] & 2014 & Non-RCT & 143 & PR plus telehealth care \\
\hline BAULDOFF [27] & 2002 & $\begin{array}{c}\text { Experimental/ } \\
\text { RCT }\end{array}$ & 24 & Walking programme \\
\hline BEHNKE [28] & 2005 & Non-RCT & 88 & Walking programme \\
\hline BendstRup [29] & 1997 & RCT & 32 & PR programme \\
\hline BERRY [30] & 2010 & RCT & 176 & $\begin{array}{l}\text { PR programme plus } \\
\text { self-management }\end{array}$ \\
\hline BoRges [31] & 2014 & RCT & 29 & Resistance training \\
\hline BREYER [32] & 2010 & RCT & 60 & Walking programme \\
\hline CASABURI [33] & 2012 & RCT & 22 & Oxygen therapy \\
\hline Coronado [34] & 2003 & Non-RCT & 15 & PR programme \\
\hline
\end{tabular}

Nutritional supplementation

Nutritional supplementation PR programme NMES

PA counselling plus PR programme

Nocturnal NIPPV plus PR programme

Nocturnal NIPPV plus physiotherapy

PR programme plus self-management PR programme

EgAN [42]

GoRIs [46]
EngSTRöm [43]

FAAGER [44]

FAULKNER [45]

HatAJI [14]

HosPEs [47]

KESTEN [48]

Kozu [49]

LARSON [50]

Mendoza [51]

Mercken [52]

Mohammadi [53]

MoY [54]

MoY [55]

Nguyen [11]

Nield [56]

Nield [57]

NinOt [58]

NishiJIMA [59]

Pitta [8]

Pleguezuelos [60]

Pomidori [61]

Probst [62]

RIES [63]

SANDLAND [64]

Sewell [65]

Sewell [66]

SHIOYA [67]

SKUMLIEN [68]

2008

2008

2003

2012

1999

2004

2010

2003

2013

2009

2008

2011

2014

2015

2005

2013

2010

2012

2009

2005

2007

2007

2015

2008

2013

2012

2011

1995

2008

2005

Steele [69]

\section{Non-RCT}

RCT

Pilot/RCT

Pilot/RCT

RCT

Pilot/non-RCT

RCT

RCT

Non-RCT

RCT

RCT

Non-RCT

RCT

Non-RCT

Pilot/non-RCT

Pilot/RCT

Non-RCT

RCT

Non-RCT

Non-RCT

Non-RCT

RCT

RCT

RCT

RCT

RCT

RCT

RCT

RCT

Non-RCT

Non-RCT

\section{7}

50

20

14

20

23

35

46

45

49

102

11

106

24

27

17

48

40

23

18

29

125

36

40

119

20

180

95

17

40

38
PR plus walking programme PR programme

PR programme plus PA counselling

Nutritional supplementation Bronchodilator

PA counselling

Bronchodilator

PR programme

Resistance training

PA counselling

PR programme

Home-based rehabilitation

PA counselling

PA counselling

PA counselling

PR programme

Breathing exercises

PR programme

Bronchodilator

PR programme

PA counselling

Walking programme

PR programme

PR programme

Oxygen therapy

PR programme

PR programme

Bronchodilator

PR programme

PR programme
PA measurement

Pedometer Yamax Digi-Walker SW-200

Nonvalidated self-reported physical activity questionnaire

Pedometer 342 and daily logs

Accelerometer Trictrac-R3D

Activities of Daily Living score specific for COPD

The Community Health Activities Model Program for Seniors

Accelerometer DynaPort Minimod

Accelerometer DynaPort Activity Monitor Accelerometer RT3

Accelerometer ADXL05

Accelerometer SenseWear Armband PRO3

Accelerometer SenseWear Armband PRO3

Pedometer NL-200 Activity Monitor Accelerometer RT3

Pedometer Yamax Digi-Walker SW-200

Pedometer Yamax Digi-Walker SW-200

Groningen Activity and Restriction Scale

Pedometer Yamax Digi-Walker SW-200

Accelerometer Sense Wear Armband PRO3 Sickness Impact Profile
Stanford Health Assessment Questionnaire

7-day physical activity recall questionnaire

Accelerometer Tracmor

Accelerometer Lifecorder

Pedometer Yamax Digi-Walker SW-200

Nonvalidated activity questionnaire

Activities of Daily Living score Actigraph 7164

Tanita PD724 pedometer

Accelerometer Physical Activity Monitor (Pam) Barthel Index

Pedometer Omron HJ-720ITC

Pedometer Omron HJ-720ITC

Accelerometer Stepwatch 3 Activity Monitor Human Activity Profile

Human Activity Profile

Physical Self Inventory-6

Lifecorder Ex 4-second version

Accelerometer DynaPort Activity Monitor Diary card

Accelerometer SenseWear Armband PRO3

Accelerometer DynaPort Activity Monitor and SenseWear Armband PRO3

Adapted form of the self-efficacy questionnaire Accelerometer Gaehwiler Z80-32k V1

Accelerometer Gaehwiler Z80-32k V1 and

Canadian Occupational Performance Measure Accelerometer Gaehwiler Z80-32k V1

London Chest Activities of Daily Living Scale

Hyrim Physical Activity Questionnaire and Glittre ADL-test
Accelerometer Tritrac R3D 


\section{TABLE 1 Continued}

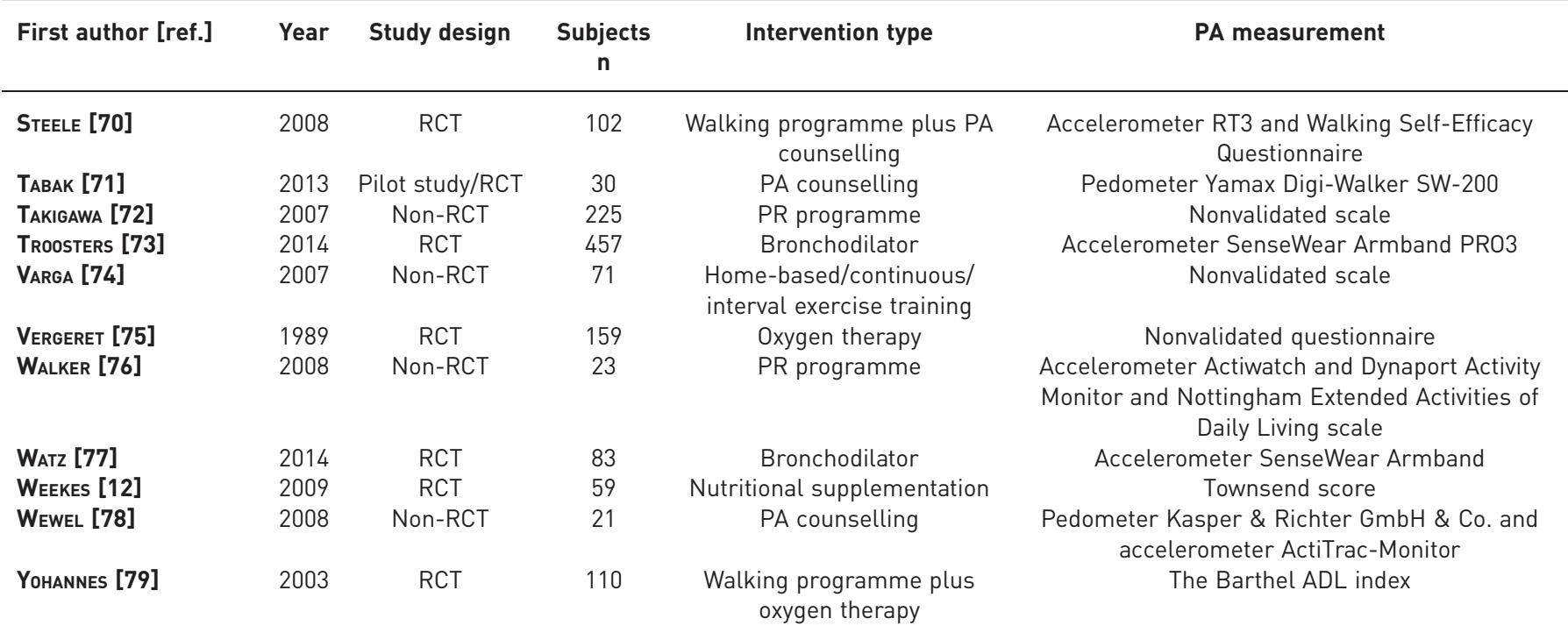

PA: physical activity; RCT: randomised controlled trial; PR: pulmonary rehabilitation; COPD: chronic obstructive pulmonary disease; NMES: neuromuscular electrical stimulation; NIPPV: noninvasive positive pressure ventilation. Additional information on the patients' characteristics, performed interventions and methods of measuring PA is presented in supplementary table S1.

\section{LTOT}

All of the studies assessing the benefits of LTOT on PA levels in patients with COPD were RCTs. Two of the three included studies used accelerometers to measure PA levels. Patients were characterised by moderate to very severe airflow limitation, with low resting room air arterial oxygen saturation measured by pulse oximetry $(<90 \%)$, and had been receiving LTOT at home over a period of $\geqslant 8$ weeks. The delivery of LTOT was diverse and included the use of ambulatory lightweight oxygen cylinders and oxygen concentrators. The use of ambulatory LTOT improved PA levels in COPD in only one out of the three studies (figure 2b).

\section{Bronchodilators}

Most of the studies of bronchodilators were RCTs including patients with moderate to very severe airflow limitation. Tiotropium and indacaterol were the most used bronchodilators and were compared to placebo or another bronchodilator. $67 \%$ of the studies measured PA levels with an objective tool and four out of the six studies delivered the interventions every day for $\geqslant 12$ weeks. As shown in the harvest plot (figure $2 b$ ), four out of the six bronchodilator studies resulted in increased PA levels in patients with COPD.

TABLE 2 Quality of evidence for interventions used to modify physical activity levels in patients with chronic obstructive pulmonary disease

\begin{tabular}{|c|c|c|c|c|c|c|c|}
\hline \multirow[t]{2}{*}{ Intervention [ref.] } & \multirow{2}{*}{$\begin{array}{c}\text { Studies } \\
\mathrm{n}\end{array}$} & \multicolumn{5}{|c|}{ Quality assessment } & \multirow[t]{2}{*}{ Quality } \\
\hline & & Risk of bias & Inconsistency & Indirectness & Imprecision & Publication bias & \\
\hline $\begin{array}{l}\text { Physical activity counselling }[10,11,25,47 \text {, } \\
\quad 51,54,55,60,70,71,78]\end{array}$ & 11 & Serious & Serious & Not serious & Serious & Strongly suspected & Very low \\
\hline Nutritional supplementation $[12,35,36,46]$ & 4 & Serious & Serious & Not serious & Serious & Strongly suspected & Very low \\
\hline Long-term oxygen therapy $[33,64]$ & 2 & Not serious & Serious & Not serious & Serious & Strongly suspected & Very low \\
\hline Bronchodilator $[14,48,59,67,73,77]$ & 6 & Not serious & Serious & Not serious & Serious & Strongly suspected & Very low \\
\hline $\begin{array}{l}\text { Pulmonary rehabilitation }[8,10,29,34,37 \text {, } \\
39,42,44,49,52,56,58,62,63,65,66 \text {, } \\
69,72,74,76]\end{array}$ & 20 & Serious & Serious & Not serious & Serious & Unsuspected & Very low \\
\hline $\begin{array}{l}\text { Other exercise training }[30,32,41,43,50 \text {, } \\
53,57,61,62,74,79]\end{array}$ & 11 & Serious & Serious & Not serious & Serious & Unsuspected & Very low \\
\hline
\end{tabular}

NIV: noninvasive ventilation; NMES: neuromuscular electrical stimulation. 


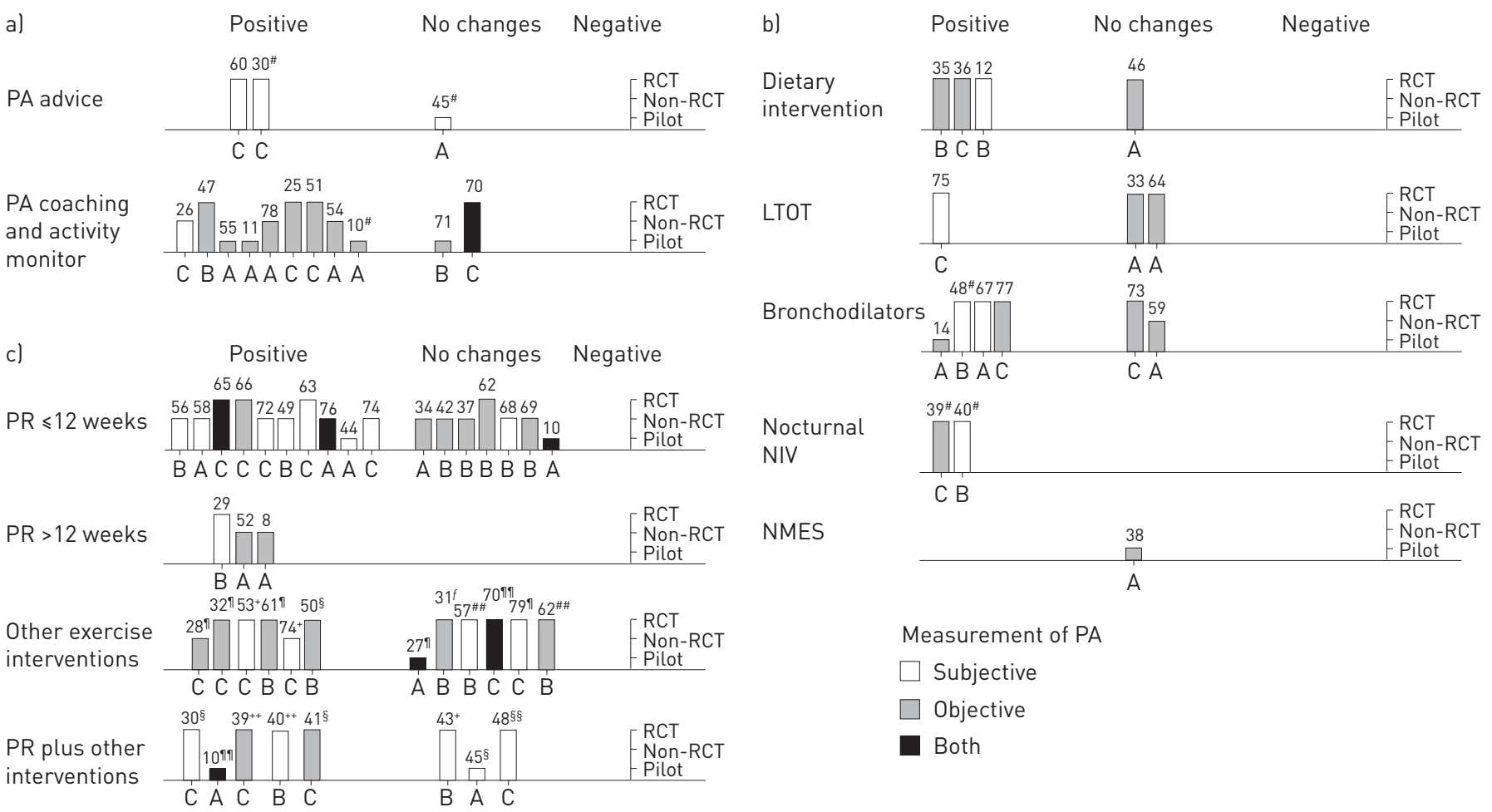

FIGURE 2 Harvest plots of the included studies. a) Studies that used physical activity (PA) counselling as the main intervention. b) Studies that used dietary intervention, long-term oxygen therapy (LTOT), bronchodilators, nocturnal noninvasive ventilation (NIV) and neuromuscular electrical stimulation (NMES) as the main intervention. c) Studies that used exercise training as the main intervention. A: studies with <30 patients; B: studies with 30-60 patients; C: studies with $>60$ patients; PR: pulmonary rehabilitation. The largest bars represent randomised controlled trials (RCTs), medium-sized bars represent non-RCTs and small bars represent pilot/experimental studies. The number on top of each bar represents the study's reference number in the present systematic review. "\#: addition of PR to the main intervention; " : walking programme; ${ }^{+}$: individualised/ home training programme; ${ }^{\S}$ : self-management/PA advice programmes; ${ }^{f}$ : resistance training alone; ${ }^{\# \#}$ : breathing exercise; ${ }^{1 \uparrow:}$ coaching programme (feedback on PA levels, individualised PA goals, and/or tailored motivational messages); ${ }^{++}$: nocturnal NIV; ${ }^{\S \S^{\prime}}$ : studies with bronchodilators.

\section{Nocturnal NIV}

Two studies assessed the impact of nocturnal NIV on PA levels in COPD patients. Both studies were RCTs and utilised exercise training in addition to the nocturnal NIV. One study assessed PA levels subjectively. Hypercapnic patients with severe and very severe airflow limitation were included in both studies. NIV was supplied via full face mask or nasal mask interface using a bilevel ventilator. Inspiratory and expiratory pressure with a spontaneous/timed mode with a backup frequency was applied. Interventions varied from 3 to 24 months' duration. All studies of nocturnal NIV in addition to exercise training increased PA levels of hypercapnic patients with COPD (figure 2b).

\section{NMES}

The only study performed in this area was a small pilot study, including eight patients with moderate to severe airflow obstruction. PA was measured with an accelerometer. NMES was applied on the quadriceps femoris of both legs through an electrical stimulation (impulses of tetanic frequency up to $19 \mathrm{~Hz}$ ) for sessions of $1 \mathrm{~h}$, 5 days per week over 8 weeks. NMES did not change daily activity levels of patients with COPD (figure 2b).

\section{$P R$ and other exercise interventions}

PR alone

One third of the 60 included studies $(n=20)$ used PR as the main intervention. $65 \%$ of the studies were non-RCTs and 11 of the 20 studies assessed PA levels with objective measurements. Most of the studies included patients with moderate to severe airflow limitation and involved high-intensity continuous exercise training (initial workload $\geqslant 60 \%$ of exercise capacity) at least twice a week over a minimum period of 7 weeks. The prescription of training intensity was based on perceived effort scales (Borg scale), heart rate and exercise capacity (work rate or maximal oxygen consumption). 13 studies showed a positive effect on PA levels in patients with COPD, while seven studies showed no change. Interestingly, all the three studies that involved $>12$ weeks of PR programme showed a positive impact on PA levels of patients with COPD (figure 2 c), while in programmes with $\leqslant 12$ weeks of training, only 10 out of 17 studies increased PA. 


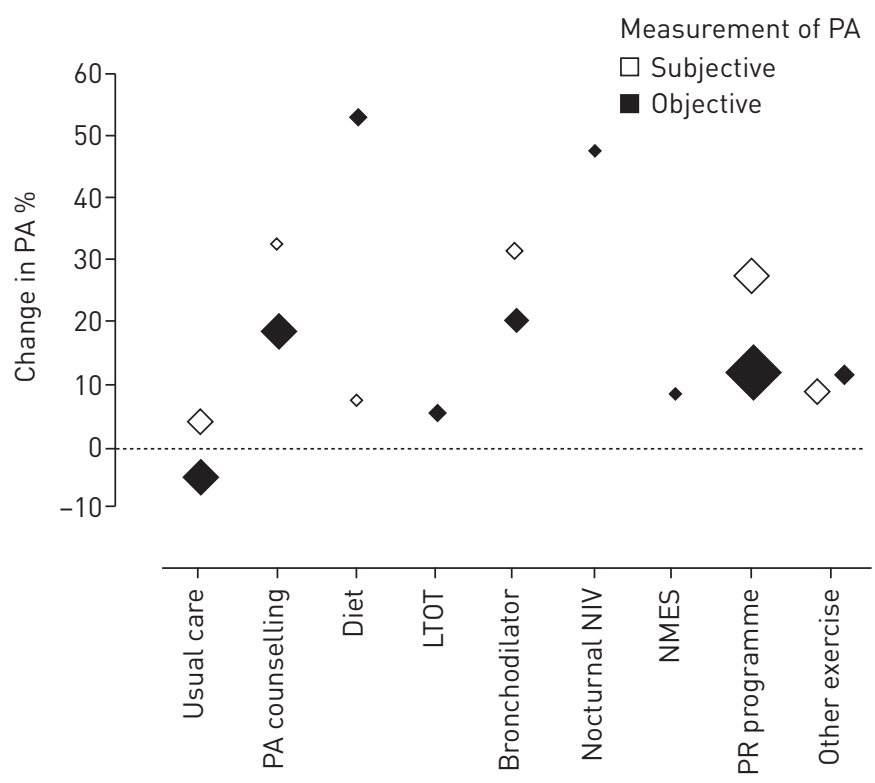

FIGURE 3 Percentage change in physical activity (PA) levels according to different intervention categories. The size of each square represents the number of studies within each category: the bigger the square, the higher the number of studies represented. Diet: dietary/nutritional intervention; LTOT: long-term oxygen therapy; NIV: noninvasive ventilation; NMES: neuromuscular electrical stimulation; PR: pulmonary rehabilitation; other exercise: other exercise programmes.

\section{Other exercise interventions}

12 studies used a wide range of exercise training including walking programmes, home training and self-management programmes (figure 2c). The majority of the interventions were based on walking exercise training, with a frequency of at least three times per week over a minimum period of 6 weeks. The intensity of the prescribed training was based on perceived dyspnoea (moderate to somewhat hard on the Borg scale), percentage of initial maximal heart rate or percentage of maximal distance/speed achieved in walking tests. Half of these studies showed no changes in PA levels in patients with COPD. This strategy was the intervention most commonly utilised in hospitalised patients.

PR combined with other exercise interventions

Eight studies combined PR programmes with other interventions. Five of these studies increased PA levels in patients with COPD (figure 2c).

\section{Effects of different interventions on daily activity levels}

Figure 3 summarises the percentage change relative to baseline in PA levels according to the seven identified categories of interventions. Overall, subjective methods showed higher percentage changes in PA in comparison to objective measurements within the same intervention category. PA counselling and bronchodilators produced similar improvements in PA levels after treatments ( $19 \%$ and $21 \%$, respectively). NMES and LTOT produced $9 \%$ and $6 \%$ increase in daily activity levels in patients with COPD, respectively. Nutritional supplementation for undernourished patients and nocturnal NIV combined with exercise training in hypercapnic patients had the biggest impact on PA levels, increasing 53\% and 48\% respectively after treatment. Exercise training programmes (PR and other exercise programmes) increased daily activity levels by $\sim 12 \%$. Unsurprisingly, usual care did not increase PA levels in patients with COPD (average decrease of $4 \%$ after treatment).

\section{Discussion}

This systematic review provides information for researchers and clinicians by identifying and summarising the broad range of interventions to modify PA levels in patients with COPD. Unfortunately, it was not possible to perform a formal meta-analysis of the outcomes due to heterogeneity of the data. Additionally, the quality of the evidence remains very low, mainly because of serious imprecision and inconsistency across studies, as well as risk of bias. 
Interventions able to modify PA levels in patients with COPD

PA in COPD patients is limited by symptoms (i.e. dyspnoea, fatigue) but also by psychosocial/behavioural factors [80]. PA coaching is a common strategy to stimulate patients towards higher levels of daily activities by modifying their behaviour. Usually, these interventions $[11,25,26,47,51,54,55,71,78]$ utilise some elements of the self-regulatory theory [81]. Using accurate assessment and feedback of PA levels, individualised PA goals and/or tailored motivational messages, patients are able to learn from successes and failures to develop an effective behavioural strategy to achieve their goal. Interestingly, PA coaching (with activity monitoring, e.g. use of a pedometer) is a very successful intervention for increasing daily activity levels of patients with COPD (figure 2a). This type of intervention is very promising and should be explored in bigger trials. Unfortunately, most of the studies in this category had a small sample size (figure 2a) $[11,47,54,55,71,78]$. Other types of PA counselling interventions, such as PA advice alone [60] or in combination with exercise training [30,45] or coaching [70], should also be further explored. To date, the results from the few available studies are still controversial and do not support a solid clinical decision $[30,45,60,70]$.

Four out of six bronchodilator studies have shown improvements in daily activity levels in patients with COPD [14, 48, 67, 77] (figure 2b). However, when considering studies using objective tools to assess PA levels, only half of the studies showed a positive impact $[14,77]$, while the other half showed no changes in PA [59, 73]. Rigorously conducted adequately powered RCTs with bronchodilators as the main intervention aiming at increasing PA levels, with objective measurement of daily activity, are required to clarify the role of bronchodilators in improving PA.

Although nutritional supplementation $[12,35,36]$ and nocturnal NIV $[39,40]$ can increase PA levels of patients with COPD, the applicability of these approaches is limited to very specific subgroups of patients. Nocturnal NIV can increase PA levels in patients with hypercapnia when combined with exercise training [39, 40]. Unfortunately, no study has explored the effect of nocturnal NIV alone. Likewise, dietary interventions produced increases in PA levels in patients with COPD with low BMI $\left(\leqslant 20 \mathrm{~kg} \cdot \mathrm{m}^{-2}\right)[12,35$, 36]. The plausibility of such interventions is poor and these results cannot be extended to the whole population of patients with COPD. However, they do show that interventions targeted at specific aspects of the disease, such as nocturnal hypoventilation in the case of nocturnal NIV and poor nutritional status in the case of nutritional supplementation, can have a positive impact on the activity levels of COPD patients. Therefore, targeting specific subpopulations of patients might be necessary in specific interventions for specific patient phenotypes. More studies in these areas are needed in order to confirm this hypothesis.

LTOT [33, 64, 75] and NMES [38] were the interventions with the lowest impact on PA levels in patients with COPD. When considering objective measurement of PA, LTOT did not produce an increase in PA levels in this population. Likewise, NMES did not change PA levels in patients with COPD [38]. Perhaps these interventions may be useful in combination with other approaches (e.g. PA coaching) aiming specifically at increasing PA. More studies are needed to explore this possibility.

As expected, exercise training was the most commonly used strategy to modify PA levels in patients with COPD. However, the effect of PR programmes to increase PA levels is still controversial. 13 studies showed an increase in PA levels $[8,29,44,49,52,56,58,63,65,66,72,74,76]$, while seven studies showed no effects after the intervention $[10,34,37,42,62,68,69]$. Interestingly, all the studies that failed to improve PA used programmes $\leqslant 12$ weeks of duration. All studies involving programmes $>12$ weeks showed a positive impact on PA $[8,29,52]$. An even higher proportion of studies with programmes $\leqslant 12$ weeks (six out of nine studies) showed no changes in PA levels when studies with objective measurements of PA were considered (figure 2c), whereas most of the studies that assessed PA with subjective tools showed positive effects of PR [44, 49, 56, $58,63,65,72,74,76]$. Since patients with COPD substantially overestimate their activity levels in daily life $[82,83]$, this finding may suggest that "short" PR programmes may impact positively on patients' perception of activities of daily living, but do not enhance their PA levels when objectively measured. In contrast, longer programmes (>12 weeks) seem to achieve better results $[8,29,52]$. Possibly, the translation of the gains (i.e. exercise capacity) of the PR programme into a more active lifestyle requires longer periods of training [8].

Other exercise interventions are also able to impact positively on PA levels of patients with COPD [10, 28, $30,32,39-41,50,53,61,74]$. Walking programmes have been commonly used to enhance PA. Walking is a usual activity for patients and is easy to perform. When considering this strategy, duration $\left(\geqslant 30\right.$ min.day $\left.{ }^{-1}\right)$ and frequency (at least three times per week over a minimum period of 3 months) of the intervention seem to be important. Similarly, the intensity of the intervention in walking programmes might be a key factor for success. When walking programmes involve activities with a high metabolic demand (i.e. $75 \%$ of maximal heart rate [32]), the results seem to be better [28, 32]. As shown in figure $2 \mathrm{c}, \mathrm{PR}$ can also cause a positive effect on daily activity levels when combined with other interventions [10, 30, 39-41]. 


\section{Percentage change in PA levels}

Although the heterogeneity of the studies precludes a meta-analysis, figure 3 summarises the effects of different interventions on activity levels in patients with COPD. This figure must be interpreted with caution, since we calculated the average of changes relative to baseline PA levels for each intervention category for illustrative purpose only, to give an impression of the magnitude of the effect we found for the different interventions. However no correction by confounding factors (such as FEV1, sex or age) was applied, due to data heterogeneity. As shown in figure 3, most interventions using objective measurements of PA were able to show 10-20\% increments in activity levels. As expected [82, 83], subjective methods overestimated PA levels when compared with objective measurements in most of the studies.

\section{Future research directions}

Despite the importance of PA in COPD, studies assessing the effect of interventions on PA levels in this population are in general of low quality. Future research should focus on well-designed trials with proper randomisation, concealment and blinding processes. Apart from poor quality of evidence, the interpretation of the current literature on the topic may also be limited by publication bias (i.e. due to high probability of unreported studies). Studies with negative or with lack of impacting results are also necessary to elucidate the effect of the interventions on PA levels in patients with COPD. In addition, only a few studies have explored the benefits of interventions during hospitalisation [31, 79]. PA levels are affected in patients with COPD during and after hospitalisation for acute exacerbation [84]. Therefore, interventions aiming at increasing PA levels during and early after exacerbations are needed. Moreover, the benefits of other interventions, such as inspiratory muscle training [85], high-intensity interval training [86], downhill walking [87] and/or nonlinear exercise training [88], should be explored. Finally, the practice of PA is likely to have, as any other lifestyle habit, a strong behavioural factor. Consequently, in order to have improvements in PA levels of patients with COPD, it may be necessary to identify and reduce the time these patients spend in sedentary behaviour [89]. Furthermore, patients with COPD need adaptive behaviour change to achieve significant and lasting results on high levels of PA in daily life [90].

In conclusion, the benefit of exercise training on PA levels is still controversial. However, longer-lasting programmes are more effective than shorter programmes in increasing PA levels in COPD and might be needed to facilitate a change in behaviour. PA coaching with the feedback of an activity monitor is a very promising intervention to enhance daily activity levels in COPD. The combination of this type of programme with exercise training, particularly with long programmes, has the potential to enhance the effects by combining the increase in capacity provided by the exercise training with the behavioural changes facilitated by PA coaching programmes. Similarly, interventions directed at potential determinants of PA, such as dietary intervention for cachectic patients and nocturnal NIV with exercise training for hypercapnic patients, may contribute to increasing PA in very specific subgroups of patients with COPD. LTOT and NMES do not seem to enhance PA in this population, but may be useful in combination with other strategies. Bronchodilators might cause a positive impact on daily activity levels of COPD patients.

\section{Acknowledgements}

The authors would like to thank Christopher J. Weir and Hilary Pinnock (both Telescot Research Programme, Usher Institute of Population Health Sciences and Informatics, The University of Edinburgh, Edinburgh, UK) for their valuable advice. We are also thankful to the EPSRC/Philips Healthcare and the College of Medicine and Veterinary Medicine of The University of Edinburgh for their support.

\section{References}

1 Garcia-Aymerich J, Lange P, Benet M, et al. Regular physical activity reduces hospital admission and mortality in chronic obstructive pulmonary disease: a population based cohort study. Thorax 2006; 61: 772-778.

2 Yohannes AM, Baldwin RC, Connolly M. Mortality predictors in disabling chronic obstructive pulmonary disease in old age. Age Ageing 2002; 31: 137-140.

3 Wen CP, Wai JP, Tsai MK, et al. Minimum amount of physical activity for reduced mortality and extended life expectancy: a prospective cohort study. Lancet 2011; 378: 1244-1253.

4 Bravata DM, Smith-Spangler C, Sundaram V, et al. Using pedometers to increase physical activity and improve health: a systematic review. JAMA 2007; 298: 2296-2304.

5 Garber CE, Blissmer B, Deschenes MR, et al. American College of Sports Medicine position stand. Quantity and quality of exercise for developing and maintaining cardiorespiratory, musculoskeletal, and neuromotor fitness in apparently healthy adults: guidance for prescribing exercise. Med Sci Sports Exerc 2011; 43: 1334-1359.

6 Pitta F, Troosters T, Spruit MA, et al. Characteristics of physical activities in daily life in chronic obstructive pulmonary disease. Am J Respir Crit Care Med 2005; 171: 972-977.

7 Spruit MA, Singh SJ, Garvey C, et al. An official American Thoracic Society/European Respiratory Society statement: key concepts and advances in pulmonary rehabilitation. Am J Respir Crit Care Med 2013; 188: e13-e64.

8 Pitta F, Troosters T, Probst VS, et al. Are patients with COPD more active after pulmonary rehabilitation? Chest 2008; 134: 273-280.

9 Cindy Ng LW, Mackney J, Jenkins S, et al. Does exercise training change physical activity in people with COPD? A systematic review and meta-analysis. Chron Respir Dis 2012; 9: 17-26. 
10 de Blok BM, de Greef MH, ten Hacken NH, et al. The effects of a lifestyle physical activity counseling program with feedback of a pedometer during pulmonary rehabilitation in patients with COPD: a pilot study. Patient Educ Couns 2006; 61: 48-55.

11 Nguyen HQ, Gill DP, Wolpin S, et al. Pilot study of a cell phone-based exercise persistence intervention post-rehabilitation for COPD. Int J Chron Obstruct Pulmon Dis 2009; 4: 301-313.

12 Weekes CE, Emery PW, Elia M. Dietary counselling and food fortification in stable COPD: a randomised trial. Thorax 2009; 64: 326-331.

13 Garcia-Aymerich J, Félez MA, Escarrabill J, et al. Physical activity and its determinants in severe chronic obstructive pulmonary disease. Med Sci Sports Exerc 2004; 36: 1667-1673.

14 Hataji $\mathrm{O}$, Naito $\mathrm{M}$, Ito $\mathrm{K}$, et al. Indacaterol improves daily physical activity in patients with chronic obstructive pulmonary disease. Int J Chron Obstruct Pulmon Dis 2013; 8: 1-5.

15 Gimeno-Santos E, Frei A, Steurer-Stey C, et al. Determinants and outcomes of physical activity in patients with COPD: a systematic review. Thorax 2014; 69: 731-739.

16 Vaes AW, Cheung A, Atakhorrami M, et al. Effect of "activity monitor-based" counseling on physical activity and health-related outcomes in patients with chronic diseases: a systematic review and meta-analysis. Ann Med 2013; 45: 397-412.

17 Wilson JJ, O'Neill B, Collins EG, et al. Interventions to increase physical activity in patients with COPD: a comprehensive review. COPD 2015; 12: 332-343.

18 Centre for Reviews and Dissemination (CRD). Systematic reviews: CRD's guidance for undertaking reviews in health care. York, CRD, University of York, 2009. Available from: www.york.ac.uk/media/crd/Systematic_Reviews.pdf

19 Liberati A, Altman DG, Tetzlaff J, et al. The PRISMA statement for reporting systematic reviews and meta-analyses of studies that evaluate health care interventions: explanation and elaboration. Ann Intern Med 2009; 151: W65-W94.

20 Caspersen CJ, Powell KE, Christenson GM. Physical activity, exercise, and physical fitness: definitions and distinctions for health-related research. Public Health Rep 1985; 100: 126-131.

21 Guyatt GH, Oxman AD, Schünemann HJ, et al. GRADE guidelines: a new series of articles in the Journal of Clinical Epidemiology. J Clin Epidemiol 2011; 64: 380-382.

22 Schünemann H, Hill S, Guyatt G, et al. The GRADE approach and Bradford Hill's criteria for causation. J Epidemiol Community Health 2011; 65: 392-395.

23 Crowther M, Avenell A, MacLennan G, et al. A further use for the Harvest plot: a novel method for the presentation of data synthesis. Res Synth Methods 2011; 2: 79-83.

24 Ogilvie D, Fayter D, Petticrew M, et al. The harvest plot: a method for synthesising evidence about the differential effects of interventions. BMC Med Res Methodol 2008; 8: 8.

25 Altenburg WA, ten Hacken NH, Bossenbroek L, et al. Short- and long-term effects of a physical activity counselling programme in COPD: a randomized controlled trial. Respir Med 2015; 109: 112-121.

26 Barberan-Garcia A, Vogiatzis I, Solberg HS, et al. Effects and barriers to deployment of telehealth wellness programs for chronic patients across 3 European countries. Respir Med 2014; 108: 628-637.

27 Bauldoff GS, Hoffman LA, Zullo TG, et al. Exercise maintenance following pulmonary rehabilitation: effect of distractive stimuli. Chest 2002; 122: 948-954.

28 Behnke M, Wewel AR, Kirsten D, et al. Exercise training raises daily activity stronger than predicted from exercise capacity in patients with COPD. Respir Med 2005; 99: 711-717.

29 Bendstrup KE, Ingemann Jensen J, Holm S, et al. Out-patient rehabilitation improves activities of daily living, quality of life and exercise tolerance in chronic obstructive pulmonary disease. Eur Respir J 1997; 10: $2801-2806$.

30 Berry MJ, Rejeski WJ, Miller ME, et al. A lifestyle activity intervention in patients with chronic obstructive pulmonary disease. Respir Med 2010; 104: 829-839.

31 Borges RC, Carvalho CR. Impact of resistance training in chronic obstructive pulmonary disease patients during periods of acute exacerbation. Arch Phys Med Rehabil 2014; 95: 1638-1645.

32 Breyer MK, Breyer-Kohansal R, Funk GC, et al. Nordic walking improves daily physical activities in COPD: a randomised controlled trial. Respir Res 2010; 11: 112.

33 Casaburi R, Porszasz J, Hecht A, et al. Influence of lightweight ambulatory oxygen on oxygen use and activity patterns of COPD patients receiving long-term oxygen therapy. COPD 2012; 9: 3-11.

34 Coronado M, Janssens JP, de Muralt B, et al. Walking activity measured by accelerometry during respiratory rehabilitation. J Cardiopulm Rehabil 2003; 23: 357-364.

35 Dal Negro RW, Aquilani R, Bertacco S, et al. Comprehensive effects of supplemented essential amino acids in patients with severe COPD and sarcopenia. Monaldi Arch Chest Dis 2010; 73: 25-33.

36 Dal Negro RW, Testa A, Aquilani R, et al. Essential amino acid supplementation in patients with severe COPD: a step towards home rehabilitation. Monaldi Arch Chest Dis 2012; 77: 67-75.

37 Dallas MI, McCusker C, Haggerty MC, et al. Using pedometers to monitor walking activity in outcome assessment for pulmonary rehabilitation. Chron Respir Dis 2009; 6: 217-224

38 Daly C, Coughlan GF, Hennessy E, et al. Effects of neuromuscular electrical stimulation on the activity levels and exercise capacity of patients with moderate to severe COPD. Physiother Ireland 2011; 32: 6-11.

39 Duiverman ML, Wempe JB, Bladder G, et al. Nocturnal non-invasive ventilation in addition to rehabilitation in hypercapnic patients with COPD. Thorax 2008; 63: 1052-1057.

40 Duiverman ML, Wempe JB, Bladder G, et al. Two-year home-based nocturnal noninvasive ventilation added to rehabilitation in chronic obstructive pulmonary disease patients: a randomized controlled trial. Respir Res 2011; 12 : 112.

41 Effing T, Zielhuis G, Kerstjens H, et al. Community based physiotherapeutic exercise in COPD self-management: a randomised controlled trial. Respir Med 2011; 105: 418-426.

42 Egan C, Deering BM, Blake C, et al. Short term and long term effects of pulmonary rehabilitation on physical activity in COPD. Respir Med 2012; 106: 1671-1679.

43 Engström CP, Persson LO, Larsson S, et al. Long-term effects of a pulmonary rehabilitation programme in outpatients with chronic obstructive pulmonary disease: a randomized controlled study. Scand J Rehabil Med 1999; 31: 207-213

44 Faager G, Larsen FF. Performance changes for patients with chronic obstructive pulmonary disease on long-term oxygen therapy after physiotherapy. J Rehabil Med 2004; 36: 153-158. 
Goris AH, Vermeeren MA, Wouters EF, et al. Energy balance in depleted ambulatory patients with chronic obstructive pulmonary disease: the effect of physical activity and oral nutritional supplementation. $\mathrm{Br} J \mathrm{Nutr} 2003$; 89: 725-731.

47 Hospes G, Bossenbroek L, Ten Hacken NH, et al. Enhancement of daily physical activity increases physical fitness of outclinic COPD patients: results of an exercise counseling program. Patient Educ Couns 2009; 75: 274-278.

48 Kesten S, Casaburi R, Kukafka D, et al. Improvement in self-reported exercise participation with the combination of tiotropium and rehabilitative exercise training in COPD patients. Int J Chron Obstruct Pulmon Dis 2008; 3: $127-136$.

49 Kozu R, Senjyu H, Jenkins SC, et al. Differences in response to pulmonary rehabilitation in idiopathic pulmonary fibrosis and chronic obstructive pulmonary disease. Respiration 2011; 81: 196-205.

50 Larson JL, Covey MK, Kapella MC, et al. Self-efficacy enhancing intervention increases light physical activity in people with chronic obstructive pulmonary disease. Int J Chron Obstruct Pulmon Dis 2014; 9: 1081-1090.

51 Mendoza L, Horta P, Espinoza J, et al. Pedometers to enhance physical activity in COPD: a randomised controlled trial. Eur Respir J 2015; 45: 347-354.

52 Mercken EM, Hageman GJ, Schols AM, et al. Rehabilitation decreases exercise-induced oxidative stress in chronic obstructive pulmonary disease. Am J Respir Crit Care Med 2005; 172: 994-1001.

53 Mohammadi F, Jowkar Z, Reza Khankeh H, et al. Effect of home-based nursing pulmonary rehabilitation on patients with chronic obstructive pulmonary disease: a randomised clinical trial. Br J Community Nurs 2013; 18: 398-403.

54 Moy ML, Janney AW, Nguyen HQ, et al. Use of pedometer and Internet-mediated walking program in patients with chronic obstructive pulmonary disease. J Rehabil Res Dev 2010; 47: 485-496.

55 Moy ML, Weston NA, Wilson EJ, et al. A pilot study of an Internet walking program and pedometer in COPD. Respir Med 2012; 106: 1342-1350.

56 Nield M, Hoo GS, Roper J, et al. Usefulness of the human activity profile, a functional performance measure, in people with chronic obstructive pulmonary disease. J Cardiopulm Rehabil 2005; 25: 115-121.

57 Nield MA, Soo Hoo GW, Roper JM, et al. Efficacy of pursed-lips breathing: a breathing pattern retraining strategy for dyspnea reduction. J Cardiopulm Rehabil Prev 2007; 27: 237-244.

58 Ninot G, Moullec G, Desplan J, et al. Daily functioning of dyspnea, self-esteem and physical self in patients with moderate COPD before, during and after a first inpatient rehabilitation program. Disabil Rehabil 2007; 29: $1671-1678$

59 Nishijima Y, Minami S, Yamamoto S, et al. Influence of indacaterol on daily physical activity in patients with untreated chronic obstructive pulmonary disease. Int J Chron Obstruct Pulmon Dis 2015; 10: 439-444.

60 Pleguezuelos E, Pérez ME, Guirao L, et al. Improving physical activity in patients with COPD with urban walking circuits. Respir Med 2013; 107: 1948-1956.

61 Pomidori L, Contoli M, Mandolesi G, et al. A simple method for home exercise training in patients with chronic obstructive pulmonary disease: one-year study. J Cardiopulm Rehabil Prev 2012; 32: 53-57.

62 Probst VS, Kovelis D, Hernandes NA, et al. Effects of 2 exercise training programs on physical activity in daily life in patients with COPD. Respir Care 2011; 56: 1799-1807.

63 Ries AL, Kaplan RM, Limberg TM, et al. Effects of pulmonary rehabilitation on physiologic and psychosocial outcomes in patients with chronic obstructive pulmonary disease. Ann Intern Med 1995; 122: 823-832.

64 Sandland CJ, Morgan MD, Singh SJ. Patterns of domestic activity and ambulatory oxygen usage in COPD. Chest 2008; 134: 753-760

65 Sewell L, Singh SJ, Williams JE, et al. Can individualized rehabilitation improve functional independence in elderly patients with COPD? Chest 2005; 128: 1194-1200.

66 Sewell L, Singh SJ, Williams JE, et al. Seasonal variations affect physical activity and pulmonary rehabilitation outcomes. J Cardiopulm Rehabil Prev 2010; 30: 329-333.

67 Shioya T, Satake M, Sato K, et al. Long-term effect of the $\beta_{2}$-receptor agonist procaterol on daily life performance and exercise capacity in patients with stable chronic obstructive pulmonary disease. Clinical study with special reference to health-related quality of life and activities of daily living. Arzneimittelforschung 2008; 58: 24-28.

68 Skumlien S, Aure Skogedal E, Skrede Ryg M, et al. Endurance or resistance training in primary care after in-patient rehabilitation for COPD? Respir Med 2008; 102: 422-429.

69 Steele BG, Belza B, Hunziker J, et al. Monitoring daily activity during pulmonary rehabilitation using a triaxial accelerometer. J Cardiopulm Rehabil 2003; 23: 139-142.

70 Steele BG, Belza B, Cain KC, et al. A randomized clinical trial of an activity and exercise adherence intervention in chronic pulmonary disease. Arch Phys Med Rehabil 2008; 89: 404-412.

71 Tabak M, Vollenbroek-Hutten MM, van der Valk PD, et al. A telerehabilitation intervention for patients with chronic obstructive pulmonary disease: a randomized controlled pilot trial. Clin Rehabil 2013; 28: 582-591.

72 Takigawa N, Tada A, Soda R, et al. Comprehensive pulmonary rehabilitation according to severity of COPD. Respir Med 2007; 101: 326-332.

73 Troosters T, Sciurba FC, Decramer M, et al. Tiotropium in patients with moderate COPD naive to maintenance therapy: a randomised placebo-controlled trial. NPJ Prim Care Respir Med 2014; 24: 14003.

74 Varga J, Porszasz J, Boda K, et al. Supervised high intensity continuous and interval training vs. self-paced training in COPD. Respir Med 2007; 101: 2297-2304.

75 Vergeret J, Brambilla C, Mounier L. Portable oxygen therapy: use and benefit in hypoxaemic COPD patients on long-term oxygen therapy. Eur Respir J 1989; 2: 20-25.

76 Walker PP, Burnett A, Flavahan PW, et al. Lower limb activity and its determinants in COPD. Thorax 2008; 63: 683-689.

77 Watz H, Krippner F, Kirsten A, et al. Indacaterol improves lung hyperinflation and physical activity in patients with moderate chronic obstructive pulmonary disease - a randomized, multicenter, double-blind, placebo-controlled study. BMC Pulm Med 2014; 14: 158.

78 Wewel AR, Gellermann I, Schwertfeger I, et al. Intervention by phone calls raises domiciliary activity and exercise capacity in patients with severe COPD. Respir Med 2008; 102: 20-26. 
79 Yohannes AM, Connolly MJ. Early mobilization with walking aids following hospital admission with acute exacerbation of chronic obstructive pulmonary disease. Clin Rehabil 2003; 17: 465-471.

80 Miravitlles M, Cantoni J, Naberan K. Factors associated with a low level of physical activity in patients with chronic obstructive pulmonary disease. Lung 2014; 192: 259-265.

81 Baumeister RF, Vohs KD, DeWall CN, et al. How emotion shapes behavior: feedback, anticipation, and reflection, rather than direct causation. Pers Soc Psychol Rev 2007; 11: 167-203.

82 Pitta F, Troosters T, Probst VS, et al. Quantifying physical activity in daily life with questionnaires and motion sensors in COPD. Eur Respir J 2006; 27: 1040-1055.

83 Pitta F, Troosters T, Spruit MA, et al. Activity monitoring for assessment of physical activities in daily life in patients with chronic obstructive pulmonary disease. Arch Phys Med Rehabil 2005; 86: 1979-1985.

84 Pitta F, Troosters T, Probst VS, et al. Physical activity and hospitalization for exacerbation of COPD. Chest 2006; 129: 536-544.

85 Charususin N, Gosselink R, Decramer M, et al. Inspiratory muscle training protocol for patients with chronic obstructive pulmonary disease (IMTCO study): a multicentre randomised controlled trial. BMJ Open 2013; 3: e003101.

86 Vogiatzis I, Nanas S, Roussos C. Interval training as an alternative modality to continuous exercise in patients with COPD. Eur Respir J 2002; 20: 12-19.

87 Camillo CA, Burtin C, Hornikx M, et al. Physiological responses during downhill walking: a new exercise modality for subjects with chronic obstructive pulmonary disease? Chron Respir Dis 2015; 12: 155-164.

88 Klijn P, van Keimpema A, Legemaat M, et al. Nonlinear exercise training in advanced chronic obstructive pulmonary disease is superior to traditional exercise training. A randomized trial. Am J Respir Crit Care Med 2013; 188: 193-200.

89 Cavalheri V, Straker L, Gucciardi DF, et al. Changing physical activity and sedentary behaviour in people with COPD. Respirology 2016; 21: 419-426.

90 Spruit MA, Pitta F, McAuley E, et al. Pulmonary rehabilitation and physical activity in patients with chronic obstructive pulmonary disease. Am J Respir Crit Care Med 2015; 192: 924-933. 